\section{Aspectos sociodemográficos associados a três comportamentos sedentários em trabalhadores brasileiros}

\author{
Socio-demographic factors associated with three \\ sedentary behaviors in Brazilian workers
}

\section{Aspectos sociodemográficos asociados a tres comportamientos sedentarios en trabajadores brasileños}

\begin{abstract}
This study focused on the association between socio-demographic characteristics and three sedentary behaviors among industrial workers in Brazil. Data were analyzed on 47,477 workers from 24 Brazilian states, collected by questionnaires from 2006 to 2008. Individual and simultaneous presence of $\geq 4$ hours of TV time per day, commuting by car or motorcycle, and sitting most of the time at work were investigated, as well as associations between these behaviors and gender, age, schooling, and family income. Simple and multiple binary logistic regression were used. Older workers and those with more schooling and higher income had increased odds of presenting two or all of the three target behaviors. Associations between gender and sedentary behaviors depend on the specific behavior. Such information can be useful for planning interventions to reduce sedentary behavior, with better targeting and more effectiveness in reaching different population subgroups.
\end{abstract}

Sedentary Lifestyle; Workers; Industry
Leandro Martin Totaro Garcia 1 Mauro Virgílio Gomes de Barros 2 Kelly Samara da Silva 3 Giovâni Firpo Del Duca ${ }^{3}$ Filipe Ferreira da Costa 4 Elusa Santina Antunes de Oliveira 2 Markus Vinicius Nahas ${ }^{3}$

\section{Resumo}

O objetivo foi investigar a associação entre aspectos sociodemográficos e três comportamentos sedentários em trabalhadores da indústria no Brasil. Analisaram-se dados de 47.477 trabalhadores das indústrias de 24 Unidades Federativas, coletados por questionário de 2006 a 2008. Analisou-se a presença individual e simultânea de assistência à televisão $\geq 4$ horas/dia, deslocamento de carro ou moto para o trabalho e passar a maior parte do tempo sentado no trabalho, assim como a associação desses comportamentos sedentários com sexo, idade, renda familiar mensal e nível de escolarização. Para tanto, utilizou-se a regressão logística binária simples e múltipla. Trabalhadores com mais idade, escolarização e renda tiveram maiores odds de apresentar dois dos três comportamentos sedentários investigados, assim como a presença simultânea deles. As associações entre sexo e comportamentos sedentários dependem do comportamento investigado. Tais informações podem ajudar no planejamento de ações de redução de comportamentos sedentários mais bem direcionadas e mais eficientes no alcance de diferentes subgrupos da população.

Estilo de Vida Sedentário; Trabalhadores; Indústria 


\section{Introdução}

Há uma crescente quantidade de achados científicos que apontam que passar tempo demasiado em atividades sedentárias possui implicações específicas e distintas para a saúde quando comparado ao fato de não se atingir os níveis recomendados de atividade física 1,2,3. Estudos longitudinais têm apontado uma relação causal entre o comportamento sedentário e a probabilidade aumentada de mortalidade por doenças cardiovasculares e por todas causas, independentemente do nível de atividade física 4,5 . Nesse sentido, a identificação de grupos populacionais com maior grau de exposição a esse tipo de comportamento é necessária, pois sob a perspectiva da causalidade social, a relação entre os padrões de morbimortalidade e fatores intermediários (materiais, psicossociais e comportamentais, como o comportamento sedentário) é moderada por aspectos sociodemográficos 6 .

De acordo com Owen et al. 2, a quantidade de achados científicos sobre os aspectos sociodemográficos associados ao comportamento sedentário ainda pode ser considerada modesta. Apesar de alguns padrões parecerem emergir, percebe-se que existem diferenças entre países quanto à contribuição dos aspectos sociodemográficos na determinação dos comportamentos sedentários 7,8,9,10,11. Isso indica que essa relação nem sempre é globalmente coerente, o que é esperado quando se consideram as peculiaridades econômicas, sociais, demográficas e culturais de cada país, assim como seu momento no processo de desenvolvimento 6 .

Especialmente em adultos trabalhadores, três domínios do cotidiano se destacam por contribuir de forma mais importante para o acúmulo do tempo sentado total: trabalho, deslocamento e lazer 12. Ademais, estudos têm apontado que os fatores correlatos, assim como a direção e a força de associação, podem depender do comportamento investigado 7,9,10,11. Sendo assim, a investigação dos fatores sociodemográficos associados a comportamentos sedentários nesses domínios é relevante para que seja possível identificar grupos mais vulneráveis às implicações de tais comportamentos. Esse tipo de informação é necessário para que diferentes instâncias do governo e da sociedade civil formulem estratégias eficientes para a mitigação de iniquidades que afetem a saúde das populações.

No Brasil, percebe-se que ainda há muitas dúvidas sobre quais grupos populacionais apresentam maior prevalência de comportamento sedentário, pois ainda são poucos os estudos sobre o tema no país 11,13,14,15. Desses estudos, somente os levantamentos a partir de 2009 do Sis- tema de Vigilância de Fatores de Risco e Proteção para Doenças Crônicas por Inquérito Telefônico (VIGITEL) 14 têm abrangência nacional, ainda assim, limitados ao uso de televisão nas capitais. Por outro lado, no país somente um estudo 11 teve como objetivo identificar a distribuição de mais de um comportamento sedentário em diferentes grupos populacionais, mas se restringiu à uma cidade.

O que se percebe então é que, apesar da crescente relevância dada aos comportamentos sedentários para a saúde pública e, por conseguinte, de identificar os subgrupos populacionais mais expostos a eles para planejar ações de redução desses comportamentos mais bem direcionadas e efetivas, poucas informações em nível nacional ainda existem sobre esses subgrupos, sobretudo quando se vai além do uso da televisão. Diante disso, o objetivo deste estudo foi investigar a associação entre aspectos sociodemográficos e três comportamentos sedentários em trabalhadores da indústria no Brasil.

\section{Método}

Trata-se de uma análise de dados secundários, derivados do inquérito Estilo de Vida e Hábitos de Lazer de Trabalhadores da Indústria, um estudo transversal conduzido de 2006 a 2008 (o momento da coleta variou entre os estados) com trabalhadores de indústrias no Brasil. Participaram da pesquisa trabalhadores de 24 Unidades Federativas (UF) do país. Os estados de Rio de Janeiro, Piauí e Sergipe não foram incluídos na investigação, pois os dados não foram obtidos no prazo estipulado para o inquérito.

No Brasil, consideram-se trabalhadores de indústrias aqueles que atuam em empresas dos seguintes setores: extrativismo mineral, transformação, serviços industriais de utilidade pública e construção civil 16. Na época, os trabalhadores dessas empresas representavam $24 \%$ dos trabalhadores formais do país, equivalendo a aproximadamente 8,5 milhões de pessoas 17 .

\section{Cálculo do tamanho amostral e processo de amostragem}

O cálculo do tamanho amostral e o planejamento da amostragem foram efetuados independentemente em cada UF. Para o cálculo do tamanho amostral, foram utilizados os seguintes parâmetros: prevalência de $45 \%$, referente à inatividade física no lazer ${ }^{18}$, erro amostral de três pontos percentuais e intervalo de $95 \%$ de confiança (IC95\%). Num segundo momento, houve acréscimo no tamanho amostral de $50 \%$ por 
conta do efeito de delineamento e, em seguida, de $20 \%$ por conta de perdas e recusas. A soma das amostras requeridas em cada UF foi igual a 52.774 trabalhadores.

Para o plano amostral, inicialmente os trabalhadores foram estratificados segundo o porte da indústria em que trabalhavam, definido de acordo com a quantidade de funcionários: pequeno (20 a 99), médio (100 a 499) e grande (500 ou mais). Em seguida, foi definida a quantidade de trabalhadores necessários em cada porte para compor a amostra, mantendo a proporcionalidade observada na população de referência. O terceiro passo consistiu em estratificar a quantidade de trabalhadores necessários para compor a amostra conforme as regiões geográficas de cada UF, também mantendo a proporcionalidade observada nas populações de referência.

Posteriormente, houve a seleção aleatória das indústrias. Em cada região geográfica das UF foram sorteadas de 10 a $50 \%$ das indústrias de cada porte, de acordo com a quantidade de indústrias existentes e a quantidade de trabalhadores necessários para compor a amostra. As indústrias que não autorizaram a participação de seus funcionários foram substituídas por outras de mesmo porte e ramo de atuação, na mesma região geográfica. Por fim, foram sorteados, de forma sistemática, os trabalhadores dentro das indústrias selecionadas.

\section{Coleta de dados}

Um questionário foi utilizado para a coleta dos dados. O instrumento foi construído tendo como base outros questionários descritos na literatura, previamente validados 19. Mesmo assim, o instrumento final do inquérito foi validado quanto ao conteúdo, lógica e clareza. Com relação à reprodutibilidade das questões sobre comportamento sedentário, os índices kappa e os coeficientes de correlação intraclasse apontaram concordância moderada a forte, variando de 0,40 (forma de deslocamento para o trabalho) a 0,77 (tempo assistindo televisão). Para as variáveis sociodemográficas, os valores de reprodutibilidade variaram de 0,9 a 1,0 19 .

Para garantir a padronização da aplicação dos questionários, os avaliadores receberam treinamento e um manual sobre os procedimentos adequados. O questionário foi aplicado em grupos de 3 a 15 trabalhadores, havendo sempre dois avaliadores por grupo. Previamente à entrega dos questionários, os trabalhadores foram informados do caráter voluntário da participação na pesquisa e da garantia de confidencialidade de suas respostas. Os dados foram tabulados por meio de leitura ótica dos questionários, realizada pelo programa Sphynx (Sphynx Software Solutions Inc., Washington DC, Estados Unidos).

\section{Variáveis do estudo}

Os seguintes comportamentos sedentários foram analisados: deslocar-se de carro ou moto para o trabalho, passar a maior parte do tempo no trabalho sentado e assistir à televisão por pelo menos quatro horas num dia habitual da semana. Também foi analisada a presença concomitante dos três comportamentos. Como variáveis independentes, foram utilizadas as seguintes informações sociodemográficas: sexo, idade, nível de escolarização e renda familiar mensal (que expressa múltiplos de um salário mínimo vigente em 2005 - R\$300,00 -, momento da construção do questionário. De 2006 a 2008, o salário mínimo passou a ser de $\mathrm{R} \$ 350,00, \mathrm{R} \$ 380,00$ e $\mathrm{R} \$ 415,00$, respectivamente). A Tabela 1 apresenta as questões, as possibilidades de resposta e as categorizações utilizadas para todas as variáveis. Na variável "forma de deslocamento para o trabalho", o uso de ônibus não foi agrupado com o uso de carro ou moto porque nem sempre configura comportamento sedentário, dado que às vezes não é possível fazer o percurso todo sentado e em geral envolve também o deslocamento a pé. Por fim, a informação sobre as UF em que ocorreu a coleta também foi utilizada, mas somente como variável de ajuste nas análises.

\section{Análise estatística}

A descrição dos dados ocorreu por intermédio de frequências absolutas e relativas e dos seus respectivos IC95\%. Utilizou-se a regressão logística binária simples e múltipla e os resultados foram expressos em odds ratio (OR), juntamente com seu IC95\%. Foi utilizada a estratégia forward para a entrada das variáveis nas análises múltiplas, sendo selecionadas somente as variáveis com valor de $\mathrm{p} \leq 0,20$ nas análises simples. Mantiveramse nos modelos múltiplos as variáveis com valor de $\mathrm{p} \leq 0,05$ ou que alteraram os $\beta$ já existentes anteriormente em pelo menos $10 \%$. Os modelos finais foram ajustados também pelas UF em que a coleta ocorreu. Testes de multicolinearidade apontaram fatores de inflação da variância de 1,01 a 1,35 (média $=1,16$ ) e tolerância de 0,74 a 0,99 , indicando ausência de colinearidade entre as variáveis independentes. Todas as análises foram realizadas no programa estatístico Stata Standard Edition, versão 11.0 (Stata Corp., College Station, Estados Unidos). 
Tabela 1

Variáveis dependentes e independentes utilizadas no estudo.

\begin{tabular}{|c|c|c|c|}
\hline Variáveis & Questões utilizadas & Opções de resposta & Categorizações adotadas * \\
\hline $\begin{array}{l}\text { Tempo assistindo à televisão num } \\
\text { dia habitual }\end{array}$ & $\begin{array}{l}\text { Quantas horas, em média, você } \\
\text { assiste à televisão num dia normal } \\
\text { da semana? }\end{array}$ & $\begin{array}{c}\text { Não assisto à televisão; Até } 1 \text { hora; } \\
2 \text { horas; } 3 \text { horas; } 4 \text { horas; } \\
5 \text { horas ou mais }\end{array}$ & $\geq 4$ horas; $<4$ horas \\
\hline $\begin{array}{l}\text { Forma de deslocamento para o } \\
\text { trabalho }\end{array}$ & $\begin{array}{l}\text { Na maioria dos dias da semana, } \\
\text { como você se desloca para } \\
\text { ir ao trabalho? }\end{array}$ & $\begin{array}{c}\text { A pé; de bicicleta; De ônibus; } \\
\text { De carro ou moto }\end{array}$ & $\begin{array}{l}\text { De carro ou moto; A pé, } \\
\text { de bicicleta ou de ônibus }\end{array}$ \\
\hline Tempo sentado no trabalho & $\begin{array}{l}\text { Suas atividades no trabalho podem } \\
\text { ser descritas como? }\end{array}$ & $\begin{array}{l}\text { Passo a maior parte do tempo } \\
\text { sentado e, quando muito, caminho } \\
\text { curtas distâncias; Na maior parte do } \\
\text { dia, realizo atividades moderadas, } \\
\text { como caminhar rápido ou tarefas } \\
\text { manuais; Frequentemente realizo } \\
\text { atividades físicas intensas }\end{array}$ & $\begin{array}{l}\text { Passar a maior parte do tempo } \\
\text { sentado; Realizar atividades } \\
\text { moderadas e intensas na maior } \\
\text { parte do dia }\end{array}$ \\
\hline Sexo & Qual o seu sexo? & Masculino; Feminino & Masculino; Feminino \\
\hline Idade & Qual a sua idade? & $\begin{array}{c}\text { Menos de } 30 \text { anos; } 30 \text { a } 39 \text { anos; } \\
40 \text { a } 49 \text { anos; } 50 \text { anos ou mais }\end{array}$ & $\begin{array}{l}\text { Em anos completos: } \\
\leq 29 ; 30-39 ; \geq 40\end{array}$ \\
\hline Nível de escolarização & $\begin{array}{l}\text { Qual o seu nível de } \\
\text { escolarização? }\end{array}$ & $\begin{array}{l}\text { Fundamental incompleto (não } \\
\text { concluiu a 8ạ série); Fundamental } \\
\text { completo (concluiu a 8ạ série); } \\
\text { Ensino médio completo } \\
\text { (concluiu a 3ạ série do 2o grau); } \\
\text { Superior completo }\end{array}$ & $\begin{array}{l}\text { Ensino Fundamental incompleto; } \\
\text { Ensino Fundamental completo; } \\
\text { Ensino Médio completo; Ensino } \\
\text { Superior completo }\end{array}$ \\
\hline Renda familiar mensal & $\begin{array}{l}\text { Qual a sua renda familiar } \\
\text { bruta mensal? }\end{array}$ & $\begin{array}{c}\text { Até } R \$ 600 ; R \$ 601 \text { a } R \$ 1.500 ; \\
R \$ 1.501 \text { a } R \$ 3.000 ; \text { Acima de } \\
R \$ 3.000\end{array}$ & $\begin{aligned} \text { Em Reais }(R \$): & \leq 600 ; 601-1.500 \\
& \geq 1.501\end{aligned}$ \\
\hline
\end{tabular}

* No caso dos comportamentos sedentários, apresentadas na seguinte ordem: 1a) desfecho de interesse e 2a) categoria de referência.

\section{Questões éticas}

O inquérito foi aprovado pelo Comitê de Ética em Pesquisa da Universidade Federal de Santa Catarina (pareceres 306/2005 e 009/2007). O Serviço Social da Indústria do Brasil, entidade parceira na realização do inquérito, autorizou esta análise de dados.

\section{Resultados}

Responderam ao inquérito 47.886 trabalhadores, vinculados a 2.775 indústrias. O retorno de questionários em cada UF alcançou, em média, 90,6\% (desvio-padrão $=8,6$ ) da amostra calculada. No processo de tabulação e conferência dos dados foram excluídos 409 questionários (0,9\% do total) que não continham resposta referente ao sexo. Dessa forma, foram analisados dados de 47.477 trabalhadores.

Os homens representavam 69,8\% (IC95\%: $69,4-70,3)$ dos trabalhadores, enquanto as mu- lheres 30,2\% (IC95\%: 29,7-30,6). Em relação à idade, 46,1\% (IC95\%: 45,7-46,6) tinham até 29 anos, 31\% (IC95\%: 30,5-31,4) tinham de 30 a 39 anos e 22,9\% (IC95\%: 22,6-23,1) tinham 40 anos ou mais. Quanto ao nível de escolarização, 18,9\% (IC95\%: 18,6-19,3) dos trabalhadores não finalizaram o Ensino Fundamental, 15,7\% (IC95\%: 15,3-16,0) completaram esse nível e 51\% (IC95\%: 50,6-51,5) e 14,4\% (IC95\%: 14,0-14,7) completaram o Ensino Médio e o Ensino Superior, respectivamente. Por fim, 32,1\% (IC95\%: 31,7-32,5) dos trabalhadores tinham renda familiar mensal de até R\$ 600, 41,4\% (IC95\%: 41,0-41,8) tinham renda de R\$ 601 a 1.500 e 26,5\% (IC95\%: 26,1-26,9) tinham renda superior à $\mathrm{R} \$ 1.500$.

Referiram assistir à televisão por pelo menos quatro horas num dia habitual 13.245 (28,1\%; IC95\%: 27,7-28,5) trabalhadores. Mulheres trabalhadoras com idade a partir de 40 anos e com renda familiar mensal acima de $\mathrm{R} \$ 1.500$ apresentaram odds de $10 \%$ a $20 \%$ menores de assistir à televisão por pelo menos quatro horas num dia habitual quando comparados aos homens, àque- 
les com idade inferior a 30 anos e com renda inferior a $\mathrm{R} \$ 600$. Os trabalhadores que finalizaram ao menos o Ensino Fundamental apresentaram odds ao menos $15 \%$ mais elevados de assistir à televisão por pelo menos quatro horas num dia habitual do que aqueles que não finalizaram esse nível (Tabela 2).

Ao se analisar a forma de deslocamento para o trabalho, 12.240 (25,9\%; IC95\%: 25,6-26,4) industriários relataram ir habitualmente de carro ou moto. As mulheres apresentaram OR $25 \%$ menor de adotar esses meios de deslocamento do que os homens. Além disso, o uso de carro ou moto tendeu a aumentar com o incremento da idade, do nível de escolarização e da renda familiar mensal (Tabela 3).

Relataram passar a maior parte do tempo sentado no trabalho 16.660 (35,3\%; IC95\%: 34,935,8) industriários. Mulheres e trabalhadores com 40 anos ou mais de idade apresentaram odds $218 \%$ e $23 \%$ superiores aos observados, respectivamente, nos homens e naqueles com menos de 30 anos de idade. Além disso, o OR de passar a maior parte do tempo sentado no trabalho tendeu a aumentar com o incremento do nível de escolarização e da renda familiar mensal (Tabela 4).
Por fim, observou-se que 1.726 (3,7\%; IC95\%: 3,5-3,9) trabalhadores apresentaram simultaneamente os três comportamentos. As mulheres apresentaram OR 17\% maior de apresentar os três comportamentos do que os homens e o OR da presença dessa tríade tendeu a aumentar com o incremento da idade, do nível de escolarização e da renda familiar mensal (Tabela 5).

\section{Discussão}

Este estudo buscou identificar aspectos sociodemográficos associados a três comportamentos sedentários, em diferentes domínios, em trabalhadores da indústria do Brasil. Os industriários brasileiros com mais idade e com maior nível de escolarização e de renda familiar mensal apresentaram OR mais elevadas de se deslocar de carro ou moto para o trabalho, de estar a maior parte do tempo sentado no trabalho e de apresentar os três comportamentos simultaneamente. Por outro lado, as associações com o sexo dependeram do comportamento investigado. Homens apresentaram OR maiores de assistir à televisão por pelo menos quatro horas num dia habitual e de se deslocar de carro ou moto para o

Tabela 2

Prevalência e odds ratio (OR) de assistência à TV por pelo menos quatro horas num dia habitual entre industriários, segundo aspectos sociodemográficos. Brasil, 2006-2008.

\begin{tabular}{|c|c|c|c|c|}
\hline \multirow[t]{2}{*}{ Variáveis } & \multirow[t]{2}{*}{$\mathbf{n}$} & \multirow[t]{2}{*}{$\%$} & \multicolumn{2}{|c|}{ OR (IC95\%) } \\
\hline & & & Bruto * & Ajustado *,** \\
\hline \multicolumn{5}{|l|}{ Sexo } \\
\hline Masculino & 9.648 & 29,3 & 1,00 & 1,00 \\
\hline Feminino & 3.597 & 25,4 & $0,82(0,78-0,86)$ & $0,81(0,77-0,84)$ \\
\hline \multicolumn{5}{|l|}{ Idade (em anos completos) } \\
\hline$\leq 29$ & 6.237 & 28,8 & 1,00 & 1,00 \\
\hline $30-39$ & 4.188 & 28,8 & $1,00(0,95-1,05)$ & $1,01(0,96-1,06)$ \\
\hline$\geq 40$ & 2.746 & 25,6 & $0,85(0,80-0,89)$ & $0,88(0,83-0,93)$ \\
\hline \multicolumn{5}{|l|}{ Nível de escolarização } \\
\hline Ensino Fundamental incompleto & 2.272 & 25,6 & 1,00 & 1,00 \\
\hline Ensino Fundamental completo & 2.084 & 28,3 & $1,15(1,07-1,23)$ & $1,16(1,08-1,25)$ \\
\hline Ensino Médio completo & 7.033 & 29,3 & $1,20(1,14-1,27)$ & $1,22(1,15-1,30)$ \\
\hline Ensino Superior completo & 1.830 & 27,0 & $1,08(1,01-1,16)$ & $1,14(1,05-1,24)$ \\
\hline \multicolumn{5}{|l|}{ Renda familiar mensal (em R\$) } \\
\hline$\leq 600$ & 4.235 & 28,4 & 1,00 & 1,00 \\
\hline $601-1.500$ & 5.620 & 29,1 & $1,04(0,99-1,09)$ & $1,03(0,98-1,09)$ \\
\hline$\geq 1.501$ & 3.271 & 26,4 & $0,91(0,86-0,96)$ & $0,90(0,85-0,97)$ \\
\hline
\end{tabular}

IC95\%: intervalo de 95\% de confiança.

* Todas as variáveis apresentaram valor de $p<0,001$ nas análises brutas e $\leq$ 0,05 nas análises ajustadas;

** Ajuste para as demais variáveis e Unidade Federativa da coleta. 
Tabela 3

Prevalência e odds ratio (OR) de deslocamento para o trabalho por meio de carro ou moto entre industriários, segundo aspectos sociodemográficos. Brasil, 2006-2008.

\begin{tabular}{|c|c|c|c|c|}
\hline \multirow[t]{2}{*}{ Variáveis } & \multirow[t]{2}{*}{$\mathbf{n}$} & \multirow[t]{2}{*}{$\%$} & \multicolumn{2}{|c|}{ OR (IC95\%) } \\
\hline & & & Bruto * & Ajustado *,** \\
\hline \multicolumn{5}{|l|}{ Sexo } \\
\hline Masculino & 8.690 & 26,3 & 1,00 & 1,00 \\
\hline Feminino & 3.550 & 24,9 & $0,93(0,89-0,97)$ & $0,75(0,71-0,79)$ \\
\hline \multicolumn{5}{|l|}{ Idade (em anos completos) } \\
\hline$\leq 30$ & 5.002 & 23,0 & 1,00 & 1,00 \\
\hline $30-39$ & 3.927 & 26,9 & $1,23(1,17-1,29)$ & $1,21(1,14-1,28)$ \\
\hline$\geq 40$ & 3.279 & 30,4 & $1,46(1,38-1,54)$ & $1,25(1,17-1,33)$ \\
\hline \multicolumn{5}{|l|}{ Nível de escolarização } \\
\hline Ensino Fundamental incompleto & 1.096 & 12,3 & 1,00 & 1,00 \\
\hline Ensino Fundamental completo & 1.110 & 15,0 & $1,26(1,15-1,38)$ & $1,27(1,15-1,39)$ \\
\hline Ensino Médio completo & 6.144 & 25,5 & $2,45(2,28-2,62)$ & $2,19(2,02-2,36)$ \\
\hline Ensino Superior completo & 3.867 & 57,0 & $9,45(8,73-10,23)$ & $5,55(5,05-6,10)$ \\
\hline \multicolumn{5}{|l|}{ Renda familiar mensal (em R\$) } \\
\hline$\leq 600$ & 1.691 & 11,3 & 1,00 & 1,00 \\
\hline $601-1.500$ & 4.264 & 22,0 & $2,22(2,09-2,36)$ & $1,94(1,81-2,07)$ \\
\hline$\geq 1.501$ & 6.179 & 49,7 & $7,79(7,32-8,28)$ & $5,01(4,65-5,39)$ \\
\hline
\end{tabular}

IC95\%: intervalo de 95\% de confiança.

* Todas as variáveis apresentaram valor de $p<0,001$ nas análises brutas e $\leq$ 0,05 nas análises ajustadas;

** Ajuste para as demais variáveis e Unidade Federativa da coleta.

Tabela 4

Prevalência e odds ratio $(\mathrm{OR})$ de passar a maior parte do tempo sentado no trabalho entre industriários, segundo aspectos sociodemográficos. Brasil, 2006-2008.

\begin{tabular}{|c|c|c|c|c|}
\hline \multirow[t]{2}{*}{ Variáveis } & \multirow[t]{2}{*}{$\mathrm{n}$} & \multirow[t]{2}{*}{$\%$} & \multicolumn{2}{|c|}{ OR (IC95\%) } \\
\hline & & & Bruto * & Ajustado *,** \\
\hline \multicolumn{5}{|l|}{ Sexo } \\
\hline Masculino & 8.843 & 26,8 & 1,00 & 1,00 \\
\hline Feminino & 7.817 & 55,1 & $3,35(3,21-3,49)$ & $3,18(3,04-3,33)$ \\
\hline \multicolumn{5}{|l|}{ Idade (em anos completos) } \\
\hline$\leq 29$ & 7.437 & 34,3 & 1,00 & 1,00 \\
\hline $30-39$ & 4.991 & 34,3 & $1,00(0,96-1,05)$ & $0,99(0,94-1,04)$ \\
\hline$\geq 40$ & 4.186 & 38,8 & $1,22(1,16-1,28)$ & $1,23(1,16-1,30)$ \\
\hline \multicolumn{5}{|l|}{ Nível de escolarização } \\
\hline Ensino Fundamental incompleto & 1.352 & 15,2 & 1,00 & 1,00 \\
\hline Ensino Fundamental completo & 1.410 & 19,1 & $1,32(1,21-1,43)$ & $1,23(1,13-1,34)$ \\
\hline Ensino Médio completo & 8.973 & 37,3 & $3,31(3,11-3,53)$ & $2,45(2,29-2,63)$ \\
\hline Ensino Superior completo & 4.890 & 72,1 & $14,4(13,3-15,6)$ & $6,99(6,39-7,66)$ \\
\hline \multicolumn{5}{|l|}{ Renda familiar mensal (em R\$) } \\
\hline$\leq 600$ & 3.036 & 20,3 & 1,00 & 1,00 \\
\hline $601-1.500$ & 6.250 & 32,3 & $1,87(1,78-1,97)$ & $1,59(1,50-1,68)$ \\
\hline$\geq 1.501$ & 7.236 & 58,2 & $5,47(5,19-5,77)$ & $3,00(2,81-3,20)$ \\
\hline
\end{tabular}

IC95\%: intervalo de 95\% de confiança.

* Todas as variáveis apresentaram valor de $p<0,001$ nas análises brutas e $\leq 0,05$ nas análises ajustadas;

** Ajuste para as demais variáveis e Unidade Federativa da coleta. 
Prevalência e odds ratio (OR) de apresentar simultaneamente os três comportamentos sedentários analisados * entre industriários, segundo aspectos sociodemográficos. Brasil, 2006-2008.

\begin{tabular}{|c|c|c|c|c|}
\hline \multirow[t]{2}{*}{ Variáveis } & \multirow[t]{2}{*}{$\mathbf{n}$} & \multirow[t]{2}{*}{$\%$} & \multicolumn{2}{|c|}{ OR (IC95\%) } \\
\hline & & & Bruto ** & Ajustado $* \star, \star \star \star$ \\
\hline \multicolumn{5}{|l|}{ Sexo } \\
\hline Masculino & 1.065 & 3,3 & 1,00 & 1,00 \\
\hline Feminino & 661 & 4,7 & $1,47(1,33-1,62)$ & $1,17(1,06-1,30)$ \\
\hline \multicolumn{5}{|l|}{ Idade (em anos) } \\
\hline$\leq 29$ & 641 & 3,0 & 1,00 & 1,00 \\
\hline $30-39$ & 557 & 3,9 & $1,31(1,16-1,46)$ & $1,22(1,08-1,37)$ \\
\hline$\geq 40$ & 523 & 4,9 & $1,68(1,49-1,89)$ & $1,39(1,22-1,58)$ \\
\hline \multicolumn{5}{|l|}{ Nível de escolarização } \\
\hline Ensino Fundamental incompleto & 50 & 0,6 & 1,00 & 1,00 \\
\hline Ensino Fundamental completo & 70 & 1,0 & $1,69(1,17-2,43)$ & $1,64(1,14-2,39)$ \\
\hline Ensino Médio completo & 832 & 3,5 & $6,30(4,73-8,38)$ & $4,91(3,65-6,59)$ \\
\hline Ensino Superior completo & 768 & 11,4 & $22,44(16,83-29,93)$ & $10,62(7,82-14,45)$ \\
\hline \multicolumn{5}{|l|}{ Renda familiar mensal (em R\$) } \\
\hline$\leq 600$ & 137 & 0,9 & 1,00 & 1,00 \\
\hline $601-1.500$ & 540 & 2,8 & $3,10(2,56-3,74)$ & $2,31(1,90-2,81)$ \\
\hline$\geq 1.501$ & 1.035 & 8,4 & $9,80(8,18-11,73)$ & $4,39(3,60-5,37)$ \\
\hline
\end{tabular}

IC95\%: intervalo de 95\% de confiança.

* Assistir à TV por pelo menos quatro horas num dia habitual, deslocar-se para o trabalho por meio de carro ou moto e passar a maior parte do tempo sentado no trabalho;

** Todas as variáveis apresentaram valor de $p<0,001$ nas análises brutas e $\leq$ 0,05 nas análises ajustadas;

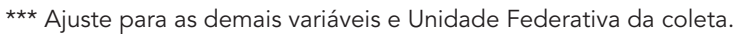

trabalho do que as mulheres. No entanto, resultado inverso foi observado quando considerado o fato de passar a maior parte do tempo sentado no trabalho e de apresentar os três comportamentos simultaneamente.

Ainda são escassas as informações sobre os fatores associados ao comportamento sedentário em adultos no Brasil 11,13,14,15, especialmente a comportamentos vinculados a diferentes domínios do cotidiano. De fato, somente um trabalho nacional anterior abordou esse tópico, analisando mais de um comportamento sedentário 11. Os demais focaram em tempo assistindo à televisão 14 , de tela 15 ou total sentado ${ }^{13}$. Ainda, alguns poucos investigaram o deslocamento por carro ou moto para o trabalho 20,21 , mas de forma secundária, uma vez que tinham como foco o deslocamento ativo. Esse cenário também se repete ao redor do mundo 7 .

Os resultados deste estudo corroboram em grande parte as associações observadas por Mielke et al. ${ }^{11} \mathrm{em}$ adultos de Pelotas (Rio Grande do Sul) (até o momento, o único no país a analisar fatores associados a distintos comportamentos sedentários); contudo, os resultados divergem quando se comparam os resultados sobre assistir à televisão em relação a sexo, idade e nível de escolarização. Por outro lado, prevalências mais elevadas de uso de televisão por mais horas entre os mais jovens foram igualmente observadas pelo VIGITEL ${ }^{14}$, que também tem escala nacional, na mesma época do nosso estudo. Dados de outros países sobre fatores sociodemográficos associados a comportamentos sedentários 7,9,10 também apontam tanto divergências como associações consistentes entre comportamentos, além de diferenças entre países.

Esse cenário indica que não é possível generalizar resultados obtidos sobre um comportamento sedentário a outros, e de que cautela é necessária quando se pretende usar evidências de uma região ou país diferente da população-alvo. Primeiro, porque, como indicam os resultados deste e de outros estudos 7,9,10,11, os aspectos correlatos podem variar de acordo com o comportamento sedentário analisado, e quando são os mesmos entre comportamentos, ainda podem haver diferenças quanto à direção e força de associação. Em segundo lugar, porque as inconsistências nos achados entre os estudos que 
investigam os mesmos comportamentos podem ser resultantes das características específicas das populações pesquisadas e das diferenças nos aspectos contextuais (sociais, econômicos, culturais etc.) entre as localidades estudadas. Por fim, deve-se ter em conta que as diferenças entre os resultados também podem estar relacionadas com a forma como as variáveis foram operacionalmente definidas, mensuradas e analisadas em cada estudo.

É muito provável que a diferença entre sexos em relação ao comportamento sedentário nos diferentes domínios do dia a dia se deva, ao menos em parte, às diferenças nos papéis social e econômico que mulheres e homens desempenham em cada sociedade, o que influencia as atividades de lazer, ocupação e deslocamento que permeiam seus cotidianos. Por exemplo, sabe-se que diferenças de gênero no mercado de trabalho são históricas. Alguns tipos de indústrias dificilmente contratam mulheres para suas funções com maior demanda física. Por outro lado, algumas funções que envolvem mais tempo sentado (como secretariado e cargos executivos) não são vistas como inadequadas para as mulheres e, portanto, há menos barreiras para que elas as assumam ${ }^{22}$. Nesse sentido, é interessante notar que o tempo sentado no trabalho foi o comportamento que contribuiu de forma mais significativa para um OR mais elevado de apresentar simultaneamente os três comportamentos sedentários entre as mulheres.

Quanto às diferenças encontradas em relação aos grupos etários, é possível que pessoas com idade acima de 40 anos tenham tido mais oportunidades de acumular bens que facilitem o envolvimento em atividades sedentárias, como veículos motorizados e certos bens duráveis para o lar. No trabalho, em geral tiveram mais tempo para progredir na carreira e assumir cargos em que as exigências físicas normalmente são menores. Outra possibilidade é que tenham sido alocados para funções menos extenuantes (ou demitidos) para que aquelas com demandas físicas mais altas fossem assumidas por pessoas mais jovens, uma vez que a redução mais significativa da aptidão e de algumas funções físicas (como a visual), que começa aproximadamente aos 30 anos de idade 23 , pode reduzir a produtividade do trabalhador dedicado à atividade de produção.

Já as diferenças verificadas entre grupos populacionais de distintos níveis socioeconômicos (tendo como proxies neste estudo o nível de escolaridade e a renda familiar mensal) devem estar baseadas em iniquidades existentes entre esses grupos, e não em decisões mais saudáveis por parte das pessoas com menores níveis. Por exemplo, pessoas em níveis socioeconômicos mais elevados têm mais facilidade de adquirir veículos motorizados, enquanto aquelas em níveis mais baixos têm maior probabilidade de utilizarem formas ativas de transporte, como a caminhada e a bicicleta 24,25 , justamente pela sua dificuldade em adquirir um veículo motorizado privado. Ademais, pessoas com menores níveis de escolarização geralmente estão envolvidas em ocupações com maiores exigências físicas 24,25 . Por outro lado, uma renda maior permite que no lar se tenha mais acesso a serviços ou tecnologias que aumentem as opções de atividades de lazer sedentário que vão além da televisão, como computador, videogame e Internet.

Algumas limitações deste estudo devem ser consideradas. Todas as medidas de comportamento sedentário foram autorreferidas. Apesar de ser uma estratégia amplamente utilizada para estudos de grande amostra (como o National Health and Nutrition Examination Survey - NHANES 26, nos Estados Unidos), os resultados podem ser influenciados por vieses de informação e por perda de acurácia sobre o comportamento em si. Além disso, não foi possível quantificar o tempo de deslocamento para o trabalho nem o tempo sentado durante a jornada laboral. Deve-se ter em conta também a possibilidade de viés de sazonalidade, devido ao fato de o período de coleta em cada estado ter sido diferente.

Em conclusão, trabalhadores com mais idade, escolarização e renda tiveram maiores odds de apresentar dois dos três comportamentos sedentários investigados, assim como a presença simultânea deles. As associações entre sexo e comportamento sedentário dependem do comportamento investigado. Identificar os grupos populacionais mais expostos a esses comportamentos, que ocupam parte importante do cotidiano das pessoas e podem ter efeitos deletérios à saúde, é uma das informações necessárias para elaborar futuras ações que objetivem reduzi-los em nível populacional. Sabe-se que estratégias de incentivo à prática de atividade física que desconsideram as peculiaridades dos subgrupos mais inativos, uniformizando de forma demasiada suas metas e ações, não são eficazes. O mesmo se pode esperar quando se prentende reduzir o comportamento sedentário. Logo, as informações coletadas por este e outros estudos que averiguem a distribuição populacional de diferentes comportamentos sedentários podem ser úteis para o planejamento de ações mais bem direcionadas e mais eficientes no alcance de diferentes subgrupos da população. 


\section{Resumen}

El objetivo fue investigar la asociación entre aspectos sociodemográficos y tres comportamientos sedentarios en trabajadores de la industria en Brasil. Se analizaron datos de 47.477 trabajadores de industrias de 24 Unidades Federativas de Brasil, obtenidas por cuestionario de 2006 a 2008. Se investigó la presencia individual y simultánea de ver televisión $\geq 4$ horas/día, desplazamiento en coche o moto para el trabajo y pasar la mayor parte del tiempo sentado en el trabajo, así como la asociación de esos comportamientos sedentarios con sexo, edad, renta familiar mensual y nivel de escolaridad. Para eso, se utilizó la regresión logística binaria simple y múltiple. Trabajadores con más edad, escolaridad y renta tuvieron mayor razón de oportunidades de presentar dos de los tres comportamientos sedentarios investigados, así como la presencia simultánea de ellos. Las asociaciones entre sexo y comportamientos sedentarios dependen del comportamiento investigado. Tales informaciones pueden ser útiles para planear acciones de reducción de comportamientos sedentarios mejor dirigidas y más eficientes en el alcance a diferentes subgrupos de la población.

Estilo de Vida Sedentario; Trabajadores; Industrias

\section{Colaboradores}

L. M. T. Garcia participou da concepção da ideia original do artigo, análise e interpretação dos dados, redação do artigo e aprovação da versão submetida. M. V. G. Barros e E. S. A. Oliveira colaboraram na concepção e execução da pesquisa, revisão crítica do artigo e aprovação da versão submetida. K. S. Silva, G. F. Del Duca e F. F. Costa contribuíram na análise e interpretação dos dados, redação do artigo e aprovação da versão submetida. M. V. Nahas participou da concepção e execução da pesquisa, concepção da ideia original do artigo, revisão crítica do artigo e aprovação da versão submetida.

\section{Agradecimentos}

Este artigo é derivado da pesquisa Estilo de Vida e Hábitos de Lazer dos Trabalhadores da Indústria, desenvolvida por meio de uma parceria entre o Serviço Social da Indústria (SESI) e o Núcleo de Pesquisa em Atividade Física e Saúde (NuPAF). A pesquisa contou com o apoio logístico e financeiro do SESI, que permitiu a utilização dos dados para fins acadêmicos e não influenciou a publicação do artigo.

\section{Referências}

1. Hamilton MT, Healy GN, Dunstan DW, Zderic TW, Owen N. Too little exercise and too much sitting: inactivity physiology and the need for new recommendations on sedentary behavior. Curr Cardiovasc Risk Rep 2008; 2:292-8.

2. Owen N, Healy GN, Matthews CE, Dunstan DW. Too much sitting: the population health science of sedentary behavior. Exerc Sport Sci Rev 2010; 38:105-13.

3. Katzmarzyk PT. Physical activity, sedentary behavior, and health: paradigm paralysis or paradigm shift? Diabetes 2010; 59:2717-25.

4. Proper KI, Singh AS, van Mechelen W, Chinapaw MJ. Sedentary behaviors and health outcomes among adults: a systematic review of prospective studies. Am J Prev Med 2011; 40:174-82.
5. Wilmot EG, Edwardson CL, Achana FA, Davies MJ, Gorely T, Gray LJ, et al. Sedentary time in adults and the association with diabetes, cardiovascular disease and death: systematic review and metaanalysis. Diabetologia 2012; 55:2895-905.

6. World Health Organization. A conceptual framework for action on the social determinants of health. Geneva: World Health Organization; 2010.

7. Rhodes RE, Mark RS, Temmel CP. Adult sedentary behavior: a systematic review. Am J Prev Med 2012; 42:e3-28.

8. Bauman A, Ainsworth BE, Sallis JF, Hagströmer M, Craig CL, Bull FC, et al. The descriptive epidemiology of sitting: a 20-country comparison using the International Physical Activity Questionnaire (IPAQ). Am J Prev Med 2011; 41:228-35. 
9. Stamatakis E, Coombs N, Rowlands A, Shelton N, Hillsdon M. Objectively-assessed and self-reported sedentary time in relation to multiple socioeconomic status indicators among adults in England: a cross-sectional study. BMJ Open 2014; 4:e006034.

10. Stamatakis E, Grunseit AC, Coombs N, Ding D, Chau JY, Phongsavan P, et al. Associations between socio-economic position and sedentary behaviour in a large population sample of Australian middle and older-aged adults: the Social, Economic, and Environmental Factor (SEEF) study. Prev Med 2014; 63:72-80.

11. Mielke GI, Silva ICM, Owen N, Hallal PC. Brazilian adults' sedentary behaviors by life domain: population-based study. PLoS One 2014; 9:e91614.

12. Chau JY, der Ploeg HP, van Uffelen JGZ, Wong J, Riphagen I, Healy GN, et al. Are workplace interventions to reduce sitting effective? A systematic review. Prev Med 2010; 51:352-6.

13. Suzuki CS, Moraes SA, Freitas ICM. Média diária de tempo sentado e fatores associados em adultos residentes no Município de Ribeirão Preto-SP, 2006: Projeto OBEDIARP. Rev Bras Epidemiol 2010 13:699-712.

14. Ministério da Saúde. Vigitel Brasil 2009: vigilância de fatores de risco e proteção para doenças crônicas por inquérito telefônico. Brasília: Ministério da Saúde; 2010

15. Gomes VB, Siqueira KS, Sichieri R. Atividade física em uma amostra probabilística da população do Município do Rio de Janeiro. Cad Saúde Pública 2001; 17:969-76.

16. Serviço Social da Indústria. Perfil do trabalhador formal brasileiro. Brasília: Serviço Social da Indústria; 2005.

17. Ministério do Trabalho. RAIS - 2006: dados estatísticos da empregabilidade no Brasil. Brasília: Ministério do Trabalho; 2007.
18. Barros MV, Nahas MV. Comportamentos de risco, autoavaliação do nível de saúde e percepção de estresse entre trabalhadores da indústria. Rev Saúde Pública 2001; 35:554-63.

19. Barros MV. Atividades físicas no lazer e outros comportamentos relacionados à saúde dos trabalhadores da indústria no estado de Santa Catarina, Brasil [Dissertação de Mestrado]. Florianópolis: Universidade Federal de Santa Catarina; 1999.

20. Silva KS, Garcia LMT, Oliveira ES, Del Duca GF, Araújo VC, Nahas MV. Fatores associados ao deslocamento ativo para o trabalho em industriários da Paraíba. Rev Educ Fís 2011; 22:265-72.

21. Silva SG, Del Duca GF, Silva KS, Oliveira ES, Nahas MV. Deslocamento para o trabalho e fatores associados em industriários do sul do Brasil. Rev Saúde Pública 2012; 46:180-4.

22. Goldin C. Understanding the gender gap: an economic history of American women. New York: Oxford University Press; 1990.

23. Duraković Z, Misigoj-Duraković M. Does chronological age reduce working ability? Coll Antropol 2006; 30:213-9.

24. Del Duca GF, Nahas MV, Garcia LMT, Mota J, Hallal PC, Peres MA. Prevalence and sociodemographic correlates of all domains of physical activity in Brazilian adults. Prev Med 2013; 56:99-102.

25. Del Duca GF, Rombaldi AJ, Knuth AG, Azevedo MR, Nahas MV, Hallal PC. Associação entre nível econômico e inatividade física em diferentes domínios. Rev Bras Ativ Fís Saúde 2009; 14:123-31.

26. Centers for Disease Control and Prevention. National Health and Nutrition Examination Survey. http://www.cdc.gov/nchs/nhanes.htm (acessado em 04/Abr/2014).

Recebido em 16/Set/2014

Versão final reapresentada em 26/Nov/2014

Aprovado em 04/Dez/2014 